\title{
Formation continue, salaires et transformations des marchés internes
}

\section{Pierre Béret}

\section{(2) OpenEdition \\ 1 Journals}

Édition électronique

URL : http://journals.openedition.org/travailemploi/4136

DOI : 10.4000/travailemploi.4136

ISSN : 1775-416X

Éditeur

DARES - Ministère du Travail

\section{Édition imprimée}

Date de publication : 30 mars 2009

Pagination : $67-80$

ISSN : 0224-4365

\section{Référence électronique}

Pierre Béret, «Formation continue, salaires et transformations des marchés internes », Travail et Emploi [En ligne], 117 | janvier-mars 2009, mis en ligne le 30 mars 2011, consulté le 19 avril 2019. URL : http://journals.openedition.org/travailemploi/4136; DOI : 10.4000/travailemploi.4136 


\title{
Formation continue, salaires et transformations des marchés internes
}

\author{
Pierre Béret (*)
}

En un quart de siècle, la formation professionnelle continue à l'initiative des employeurs (FPCE) s'est considérablement transformée, aussi bien du point de vue de ses caractéristiques que de ses conséquences salariales. En ne considérant que les cinq années précédant les enquêtes FQP pour 1977, 1985, 1993 et 2003, le taux de formés a explosé, la certification s'est effondrée et les durées ont fondues. De même, les conséquences liées à la conception de la formation comme investissement n'ont plus lieu d'être puisque diplôme et durée des FPCE n'ont plus d'effets allant dans ce sens. L'ancienneté dans la firme, dont l'effet n'était plus significatif en 1993, le redevient en 2003 mais seulement pour les salariés à qui l'entreprise a octroyé une formation continue, bien que cette dernière soit de faible durée (moins de 41 heures pour les trois quarts d'entre eux). Ceci s'explique par les transformations du marché du travail dans les années 1990 et les nouvelles formes organisationnelles qui émergent. Il en découle aussi, en 2003, un statut nouveau des FPCE et des mobilités au sein des marchés internes dont la traduction salariale se renforce.

Depuis une trentaine d'années, sous la pression de la concurrence internationale et de la crise qui touchent les pays industrialisés, s'opèrent de véritables mutations des organisations productives. Celles-ci se traduisent, notamment, par une demande accrue de flexibilité technique et organisationnelle, avec, pour corollaire, une transformation des modes de gestion des salariés et de nouvelles exigences sur leurs capacités.

Une des clefs de la flexibilité requise du système productif est à rechercher dans l'articulation entre un système de formation initiale et continue et un système d'emplois qui se transforment. L'enjeu, pour la formation initiale, est de produire conjointement des connaissances opérationnelles dans l'emploi (de manière à raccourcir le délai d'utilisation des enseignements reçus et d'en accroître l'efficacité productive), et des savoirs plus généraux permettant aux futurs actifs de développer une aptitude à l'autonomie et à la polyvalence. Quant à la formation professionnelle continue, elle devrait favoriser, en cours de vie active, aussi bien un élargissement qu'une conversion des savoirs, et leur compatibilité avec les transformations productives qui ont cours.

En lien avec la question des effets des politiques de formation, c'est à l'étude de cette articulation que l'on souhaite consacrer ce papier en nous focalisant sur la formation professionnelle à l'initiative des employeurs (FPCE). Cette dernière n'est cependant pas, pour l'entreprise, indépendante de ses

* Aix-Marseille Université, CNRS-LEST UMR 6123. pierre.beret@univmed.fr conditions internes d'utilisation. Pour comprendre celles-ci, il faut examiner la façon dont s'articulent la production de compétences dans le système de formation initiale et les modes de construction de la qualification et de valorisation professionnelle sur le marché du travail. Cette nécessité tient au fait que la FPCE n'est qu'un des éléments qui concourt à l'augmentation des capacités productives des agents. En effet, son efficacité, voire son existence même, dépendent aussi bien des attributs professionnels antérieurs des individus, en termes de formation et d'expérience, que des besoins en compétences des firmes auxquels la formation continue apporte une réponse.

La rationalité des acteurs économiques s'enracine dans un contexte institutionnel auquel elle ne peut échapper. Dès lors, ce sont les formes générales de structurations de l'espace de qualification qui déterminent le registre d'utilisation de la formation continue par les entreprises et les conditions de son accès et de ses effets. L'analyse des logiques à l'œuvre dans la construction des diplômes au sein du système éducatif français amène à formuler l'hypothèse que les transformations importantes des marchés du travail, en lien avec celles du système scolaire en France, ont modifié la place et le rôle de la FPCE. Dans les années 1960, les actifs n'étaient pourvus que d'un faible niveau de formation initiale, et la formation professionnelle continue avait pour fonction de contribuer à la construction des qualifications au sein des marchés internes d'entreprise. La forte augmentation de la scolarisation initiale depuis le début des années 1980 ne s'est pas pour autant accompagnée d'une transformation structurelle de 
cette offre de formations, qui reste principalement axée sur un principe de révélation de potentiels d'aptitudes individuelles. De ce fait, on peut avancer l'hypothèse qu'une fonction de tri et de sélection régit, au début des années 1990, maintenant de façon prédominante l'organisation des parcours internes aux entreprises. L'objectif sous-jacent est de repérer les salariés les plus performants et de leur favoriser l'accès à la formation continue, laquelle se comprend à la fois comme conséquence du succès de la sélection et comme pivot du système d'incitation autrefois plus articulé autour de l'avancement à l'ancienneté. Dans cette optique, la FPCE se conçoit moins comme une accumulation de capital humain que comme la reconnaissance, de fait, de compétences acquises antérieurement. Cette approche a déjà fait l'objet d'un travail antérieur (BÉRET, DUPRAY, 1998). On se propose dans cet article de le poursuivre jusque dans les années 2000. Les évolutions survenues dans les années 1990 sur les marchés du travail et la gestion des entreprises ont sensiblement changé ces caractéristiques antérieures.

Dans une première partie, après un bref panorama des travaux sur les transformations des marchés $\mathrm{du}$ travail et celles sur la formation professionnelle continue, nous présenterons nos hypothèses, déjà évaluées antérieurement pour les années 1977 et 1993, et nous préciserons celles attendues dans les années 2000. Le problème que nous voulons traiter est celui des évolutions dans la construction des qualifications, ou des compétences, sur les marchés du travail, appréhendés principalement au travers des marchés internes, et notamment, d'analyser les transformations de la place et des effets de la FPCE. Outre cette dernière, ceci implique de prendre conjointement en compte trois ensemble de déterminants qui se transforment. Les changements de la formation initiale et ceux des marchés du travail d'un double point de vue. D'une part, le rôle et la place de l'ancienneté dans l'entreprise, qui est l'une des dimensions qui concourt aux apprentissages, et à leur rémunération, au sein des marchés interne. D'autre part, au-delà du seul temps passé dans la firme, l'ensemble des mobilités internes (et externes pour les nouveaux arrivants) qui, elles aussi, témoignent de la manière dont s'élabore l'augmentation des qualifications au travers des promotions par exemple.

Une deuxième partie présentera les évolutions des effets de la FPCE sur un quart de siècle, en prenant notamment en compte la durée et la certification de ces dernières. On mènera conjointement une réflexion sur les transformations des marchés internes telles qu'on peut les appréhender au travers de l'ancienneté. Dans une troisième partie, nous interrogerons la place de la formation continue en lien avec les transformations des mobilités sur les marchés internes (promotions, changements de poste) et externes (changements d'entreprise avant les cinq années du poste actuel ou pendant ce laps de temps) pour les années 1993 et 2003.

\section{Examen des travaux sur les marchés du travail et la formation continue et hypothèses de travail}

Un des faits les mieux établis en économie du travail est relatif au rôle central des marchés internes après la Seconde Guerre mondiale. Au plan théorique, le marché interne est caractérisé par une propriété décisive, au-delà de la longue durée de la relation d'emploi qui n'en est qu'une des expressions. Il faut que les deux agents - le salarié et l'entreprise - y trouvent un intérêt économique. Pour le salarié, il s'agit évidemment du paiement d'un salaire plus élevé que celui qu'il pourrait se procurer dans une autre firme. Pour l'entreprise, il s'agit de mobiliser sa main-d'œuvre et/ou de pouvoir disposer de qualification qu'elle ne peut trouver ailleurs.

\section{Les transformations des marchés du travail}

L'objet de ce texte n'est bien sûr pas d'entrer dans les très nombreuses conceptualisations de ces marchés internes. On peut cependant d'un mot rappeler que l'approche initiale de ces marchés a été celle de la segmentation de M. Piore (avec la notion de qualification spécifique) et celle du capital humain de G. Becker (et la notion de capital spécifique). Au-delà des oppositions fondamentales entre ces deux théories, leur point commun est celui, mais pour des raisons différentes, d'une valorisation salariale de l'ancienneté des salariés dans l'entreprise.

De ce point de vue, les marchés internes sont particulièrement importants en France dans les années 1970 (Maurice, Sellier, Silvestre, 1982). À l'époque, la France conjuguait une faible scolarisation de la population avec un processus de sélection scolaire basée sur une forte hiérarchie des niveaux d'enseignement général. Ceci entraînait une absence de connaissances professionnelles pour une majorité de jeunes et une difficulté pour des entreprises à apprécier l'apport productif des formations professionnelles pour ceux en possédaient une. Le fonctionnement des marchés internes s'organisait donc autour de la construction des qualifications professionnelles des salariés à l'intérieur des firmes (Silvestre 1986; Eyraud, Marsden, Silvestre, 1990). Cette construction des qualifications par l'apprentissage sur le tas a entrainé la règle de rémunération à l'ancienneté. Ainsi, par exemple, en 1978 pour l'industrie, l'ancienneté payait deux fois plus en France qu'en Allemagne (Depardieu, PAYEN, 1986).

Dans les années 1980, un ensemble de facteurs se cumule et tend à déstabiliser les marchés internes en France (GERME, 2001). Tout d'abord, ce pays a connu une véritable révolution scolaire qui l'a amenée au tout premier rang du point de vue de la scolarisation de sa population. Cependant, les logiques de 
reconnaissance des formations restent largement basées sur la hiérarchie des niveaux d'enseignement général et la réussite aux épreuves académiques malgré le développement important de l'enseignement professionnel (VERDIER, 1995). Dans ce cadre, même si les individus sortent de formations professionnelles rénovées et de meilleur niveau, une forte incertitude demeure sur leurs capacités productives et ce, pour au moins deux raisons: d'une part l'hétérogénéité des publics, jusqu'au niveau bac inclu, s'est accrue; d'autre part, une insuffisante connaissance des employeurs de l'apport intrinsèque de la formation persiste. Dans ce cadre, le diplôme aurait d'abord un rôle allocatif et les individus progresseraient ensuite en fonction de leurs capacités réelles. Mais l'espace des mobilités possibles c'est lui-même beaucoup modifié: diminutions des lignes hiérarchiques, diminutions des mobilités promotionnelles au profit des mobilités horizontales liées à l'extension de la polyvalence, avec, finalement, un coup de frein sur les carrières (Goux, 1991). Dans ce cadre, on peut avancer l'hypothèse que les individus étant maintenant formés, ceci n'oblige plus les firmes à le faire de manière systématique. Il y a donc moins nécessité de construire des compétences internes rémunérées par le principe de l'avancement salarial à l'ancienneté: ainsi, on a constaté une nette diminution des conséquences salariales de l'ancienneté qui n'est plus significative en 1993 (BÉRET, DupraY 1998), ce qui rejoint des résultats obtenus par ailleurs (BÉret, 1992; Gautié, 2004 ; Goux, Maurin, 1994).

Dans les années 1990, deux transformations majeures ont eu lieu. La première est relative à la mise en place, dans de nombreuses entreprises, d'une gestion des compétences qui se substitue, ou s'articule, à l'antérieure gestion des qualifications (BÉRET, LEWANDOWSKI 2007). En corollaire, on assiste à une individualisation très importante des rémunérations, ces deux phénomènes renvoyant d'ailleurs à un ensemble de problèmes ou de paradoxes importants (LATTES et al., 2007). Ainsi, entre 1992 et 1998, plus de la moitié des établissements présents aux deux enquêtes REPONSE ont modifié leurs pratiques salariales à l'égard des salariés non-cadres et cadres, et le nombre d'établissements n'accordant que des augmentations individualisées a plus que doublé (BARREAU, BROCHARD, 2003). Ainsi, en 2002, huit salariés sur dix ont vu leur salaire augmenter, mais seulement $30 \%$ de ces derniers ne le doivent qu'à des augmentations générales au sein de l'entreprise (BRIZARD, 2004). La deuxième est relative au contexte dans lequel ont eu lieu ces changements. Tout d'abord, du point de vue des mobilités internes aux entreprises, entre les années 1990-2000, les travaux montrent une diminution assez sensible de la part des promotions réalisées via le marché interne au profit des promotions réalisées via un changement d'entreprise (GERME, 2001). Ceci renvoie notamment à la situation économique, puisqu'en 2000, avec le retour de la crois- sance, les mobilités entre entreprises (sans passage par le chômage) sont passées de 1,3 million de salariés entre mars 1993 et mars 1994 à 2,3 millions entre mars 2000 et mars 2001 (Amossé, 2002). Ces deux transformations correspondent aussi à des recompositions dans la structure du marché du travail et des politiques des entreprises. Ainsi, le marché secondaire (au sens de M. Piore) représentait $14 \%$ des emplois au début des années 1980, il en représente $34 \%$ en 2001 , dont la moitié correspond à l'émergence d'un marché secondaire «stable» dans lequel l'ancienneté moyenne est de plus de dix ans contre moins d'un an sur le marché secondaire «traditionnel» (VALETTE, 2007). De même, si l'on considère les politiques de gestion de l'emploi des entreprises en 1998, plus de la moitié des salariés sont dans des établissements qui mettent en œuvre «des politiques actives» qui se traduisent par des politiques salariales volontaristes (nombreuses augmentations) souvent individualisées et un haut niveau des dépenses de formation continue avec, en parallèle, une accentuation de la diminution des lignes hiérarchiques pour la plus grande partie des salariés bénéficiant de ces politiques (PETIT, 2003). On se retrouve donc devant une triple conjonction. Un fort marché secondaire (un tiers des salariés) dont l'ancienneté est faible ou bien élevée mais ne payant peu ou pas. Parallèlement, des politiques de gestion actives pour la majorité des actifs occupés avec la mise en place de démarches compétences qui se traduisent, notamment, par une individualisation des rémunérations. Si ceci implique une hétérogénéité accrue entre les salariés (ils n'avancent pas salarialement au même rythme), cela se passe néanmoins dans un système où tous en profitent (mais plus inégalitairement). Enfin, de fortes mobilités d'emploi à emploi sur le marché externe qui laisse penser à une compétition plus forte des firmes pour capter une main-d'œuvre jugée compétente. De cet ensemble de transformations, on peut avancer l'hypothèse d'un effet retrouvé de l'ancienneté.

\section{Les travaux sur la formation professionnelle continue en France}

En France, les travaux sur la formation continue (FPCE) sont de plus en plus nombreux. Ils se développent à partir de la loi de 1971 qui fait obligation légale aux entreprises de consacrer une part de leur masse salariale à la formation des salariés. Jusqu'à la fin des années 1990, les travaux statistiques sur ce thème reposaient avant tout sur les enquêtes FQP (1970, 1977, 1985, 1993) de l'Insee, sur des traitements des déclarations 24-83 des entreprises, et sur les enquêtes européennes CVTS. Les enquêtes FQP distinguent les formations «à l'initiative de l'entreprise» (c'est-à-dire financées ou organisées par l'employeur) des «formations postscolaires à l'initiative de l'individu». Par ailleurs, en 2000, une enquête portant sur la formation continue a été réalisée en complément de l'enquête Emploi de 
l'Insee. Force est de constater que la quasi-totalité des travaux sur la formation portent sur les formations des salariés «à l'initiative de l'employeur». Ceci est évidemment en lien avec l'obligation légale faite aux entreprises. Deux grands types de questionnements, qui peuvent être traités ensemble ou séparément, structurent les analyses: les taux d'accès à la formation et les effets de cette dernière.

Sur le premier point, l'objet est le plus souvent celui des inégalités, ou de leurs évolutions, dans l'accès à la FPCE, qu'il s'agisse de comparer des pays européens (Aventur, MöBus, 1996; THÉRY et al., 2002), du statut professionnel (FourNiER et al., 2001), des trajectoires précaires (PEREz, ThOMAS, 2006), des hommes et des femmes (Fournier, 2001), des jeunes ou des adultes âgés (FourNIER 2003, GÉlOt, MinNi, 2002), des catégories socioprofessionnelles, des secteurs ou des tailles d'entreprises (BentaBet et al., 2001, Perez 2003), ou de la place de l'encadrement intermédiaire (GÉLOT, 2006), de la récurrence des passages en formation (Trautmann, 2003), des diplômes (Béret, Dupray 2000A, Goux, Maurin, 1997), du type de formation suivi (HANCHANE, LAMBERT, 2003). Le constat général est celui de grandes disparités dans l'accès à la formation, disparités qui tendent globalement à perdurer.

Sur la question des effets de la FPCE à l'initiative de l'employeur, qui nous intéresse ici très directement, deux grands types de travaux existent.

Un premier ensemble analyse les conséquences de la formation à partir de la manière dont elles sont perçues par les salariés, qu'il s'agisse des effets sur le poste, la classification, le travail ou le salaire (CROCQUEY, 1995), ou sur les promotions professionnelles ascendantes et les changements de catégorie sociale et leurs évolutions (DuBAR, PoDEvin, 1990, MÉHAUt 1996). Le constat est ici celui d'un effondrement des effets de la FCPE entre les années 1970 et 1990 .

Un deuxième ensemble concerne l'estimation des effets de la FPCE sur le salaire. On peut distinguer deux cas:

-D'une part, les travaux qui estiment les effets de la FPCE en contrôlant l'hétérogénéité non observée (BÉret, Dupray 2000a, Goux, Maurin, 1997) ou en mettant l'accent sur les liens entre FC, mobilité et salaire (Dupray, Hanchane, 2001, FougÈre et al., 2001, Goux, Maurin, 1997), ou sur le financeur et l'initiateur (Dupray, Hanchane, 2003). Le constat est celui d'un impact faible (voire nul) de la FPCE sur le salaire, tout au plus cette dernière est un élément fort de maintien dans la firme, et lorsque tel n'est pas le cas, la formation atténue la perte salariale d'un changement d'employeur, tandis que les gains sont plus forts lorsque le salarié intervient dans la décision d'une formation que finance l'employeur.
-D'autre part, les travaux qui se focalisent sur la durée et la certification de la formation, ainsi que leur nombre et leurs rapports avec les promotions (BÉret, Dupray, 1998a). Le constat est ici celui d'un basculement d'une FPCE de type capital humain dans les années 1970, à une formation avant tout conçue comme un signal (en 1993, la durée et la certification ont un effet négatif sur le salaire) dont seul le nombre fait avant tout valeur. De même, la dernière FPCE a lieu plus souvent après une promotion, son rendement est plus élevé et les salariés concernés ont suivi plus de FPCE que les autres. Ainsi par exemple, la promotion au passage cadre ne dépend que très secondairement du fait d'avoir suivi une formation (GADÉA, TRANCART, 2003), par contre, le fait pour un cadre de n'avoir pas eu plusieurs formations durant les dernières années est salarialement préjudiciable (BÉRET, DUPRAY, 2000b).

Ce qui semble central, c'est donc d'intégrer le constat que si une formation donnée n'a que peu d'effets par elle-même, cela tient au fait qu'elle s'inscrit dans une trajectoire, c'est-à-dire dans une histoire professionnelle, qu'elle jalonne mais qui la détermine en retour. Les analyses précédentes montrent que ce n'est pas une FPCE qu'il faut considérer, mais l'ensemble des formations (et certaines autres caractéristiques de la trajectoire) qui sont maintenant au cœur de la construction des compétences professionnelles. La formation va à la formation, et c'est dans ces cas qu'elle est la plus efficace, tout autant parce qu'elle augmente les capacités des agents que parce qu'elle est un révélateur d'un ensemble d'autres attributs (à l'exemple de l'intervention du salarié dans la décision de formation qui atteste de son engagement professionnel).

\section{Propositions d'analyse}

On l'aura compris, l'objet de ce papier est d'essayer d'articuler trois éléments, en évolutions, qui concourent à la construction des qualifications ou des compétences, et à leur valorisation salariale sur vingt-cinq ans: la formation initiale, les marchés du travail et la FPCE. Il s'agit donc d'articuler les constats avancés ci-dessus. Les deux parties suivantes seront consacrées à l'opérationalisation de nos hypothèses d'un double point de vue. Dans un premier point, en nous focalisant sur le temps passé dans l'entreprise, et, dans un deuxième point, en prenant en compte les divers mouvements (promotions, etc. ) qui ont eu lieu sur ces mêmes marchés internes.

Dans les années 1970, le fonctionnement des marchés internes s'organisait autour de la construction des qualifications professionnelles des salariés à l'intérieur des firmes. Cette construction des qualifications par l'apprentissage sur le tas répondait à l'exigence de pénurie en main-d'œuvre qualifiée. Dans ce contexte, la FPCE renvoyait à une logique d'investissement en formation destinée à suppléer 
le manque de formation professionnelle initiale des salariés, tout en les retenant dans l'entreprise par un salaire supérieur (ce qui rendait aussi difficile des mobilités en provenance d'autres firmes ayant la même logique).

Dans les années 1980, on a avancé l'hypothèse que le diplôme avait d'abord un rôle allocatif et que les individus progressaient ensuite en fonction de leurs capacités réelles. D'ailleurs, la FPCE dont on attendait des conséquences sur la promotion sociale dans les années 1970, semble avoir fait place à la poursuite d'objectifs d'adaptation au poste et de gestion de l'emploi (Berton, Podevin, 1991). Dans ce contexte, la FCE ne ferait que ratifier le succès relatif du salarié dans l'épreuve de sélection à laquelle renvoie prioritairement l'organisation des parcours internes. La promotion aurait un rôle semblable, objectivant par le signal communiqué la reconnaissance par l'entreprise des compétences du salarié. Pour avancer sur ces points, on a examiné les effets salariaux de la FPCE des enquêtes FQP 1977 et 1993. Parallèlement à la formation continue, il convenait de prendre en compte les autres dimensions représentatives de la capacité productive de l'individu notamment la formation initiale, l'expérience professionnelle et l'ancienneté. Pour analyser la valorisation de ces éléments, on a utilisé des fonctions de gains classiques (du type de celles proposées par Mincer, Jovanovic, 1981) exposées dans l'encadré 1.

Ces hypothèses ont été largement validées sur l'ensemble de la population salariée (BÉRET, DUPRAY, 1998):

- Une baisse des effets salariaux de l'ancienneté sur les marchés internes.

- La non-valorisation du diplôme de FPCE, et le fait que plus les formations sont longues et moins elles sont rentables, ce qui rompt avec la logique classique d'accumulation du capital humain.

- Le constat que c'est la répétition des passages en FPCE qui fait avant tout valeur à la fin des années 1980.

Que peut-on dire de ces transformations dix ans après, en utilisant des données similaires issues de l'enquête FQP 2003? Pour la formation initiale, le paysage ne s'est pas fondamentalement modifié par rapport à 1993, du point de vue des flux, puisque le taux de bacheliers ou celui de l'accès à l'enseignement supérieur sont globalement stables depuis lors (mais $38 \%$ des salariés ont un diplôme égal ou supérieur au baccalauréat, soit 8 points de plus qu'en 1993, tableau 1). Par contre, on l'a vu, de considérables bouleversements ont eu lieu du point de vue des marchés du travail et des politiques de gestion des entreprises. Ainsi, globalement, on peut anticiper des effets salariaux positifs de l'ancienneté dans un cadre où émerge un marché secondaire stable important et où une part majoritaire des individus profite d'une gestion salariale active, souvent

\section{Encadré 1 \\ Définition des populations, enquêtes, variables et modèles utilisés}

Salariés hommes et femmes occupés en $1977,1985,1993$ ou 2003 et qui étaient salariés occupés en 1972, 1980, 1988 ou 1998. Les salariés ayant changé d'entreprise entre les deux dates ne sont pas exclus du champ. Remarquons que les structures des populations en termes d'âge et d'expérience professionnelle sont proches aux quatre enquêtes (tableau 1), ce qui met à l'abri d'un effet de composition des populations pour expliquer la nature des résultats. Les enquêtes de l'Insee mobilisées sont les enquêtes FQP aux quatre dates. Les effectifs réels sont présentés dans le tableau 1. Les résultats prennent en compte la pondération pour les tableaux suivants.

La FPCE est la formation suivie à l'initiative de l'employeur. II s'agit de la plus importante en 1977 et 1985; et de la dernière en 1993 et 2003. Dans tous les cas, on ne prend en compte que les FPCE terminées ayant eu lieu dans l'intervalle de cinq ans précédant les enquêtes. On dispose aussi du diplôme et de la durée de la formation continue, ainsi que du nombre total de formations suivies durant toute la vie active (pour 2003, il s'agit du nombre de formations continues de toutes natures sur les cinq dernières années). Les résultats concernent les FPCE achevées au cours des périodes 1972-1977, 1980-1985, 1988-1993 et 1998-2003.

II est par ailleurs possible de connaître l'existence d'un changement de poste ou d'une promotion obtenue chez l'employeur courant indépendamment de la question précédente sur la formation continue pour les années 1993 et 2003.

Les modèles testés sont des fonctions de gains du type:

Log $\mathrm{Wi}=$ cte $+\sum \mathrm{dk}$ DIPik + b1 EXPi + b2 EXPi2 $+\mathrm{c} 1 \mathrm{ANCi}+\mathrm{c} 2 \mathrm{ANCi} 2+\sum \mathrm{dj} F \mathrm{PCEij}+\mathrm{Ui}$

avec:

W: salaire annuel; DIP: diplôme de formation initiale. EXP: expérience professionnelle «réelle» c'est-à-dire entre 1977 ou 1993 et le premier emploi.

ANC: ancienneté dans l'entreprise de 1993: l'effet salarial d'une année passée sur le marché du travail correspond à la somme des effets de l'expérience et de l'ancienneté. FPCE: formation professionnelle continue à l'initiative de l'employeur.

dk: le coefficient de régression pour le diplôme de modalité k, k=\{aucun,cep,Cap/Bep/Bepc,Bac,Sup\}.

dj: le coefficient correspondant à la participation ou non à la FPCE; j prend deux ou trois modalités selon que l'on considère ou non si la FPCE a été certifiée.

On peut aussi introduire dans le modèle les autres variables décrites ci-dessus à la place ou en combinaison avec la FPCE. 
Tableau 1: Structure des populations et caractéristiques de l'accès à la formation continue

\begin{tabular}{|c|c|c|c|c|}
\hline & 1977 & 1985 & 1993 & 2003 \\
\hline Effectifs non pondérés & 19882 & 17888 & 8051 & 18302 \\
\hline $\begin{array}{l}\text { Structures des populations: } \\
\text { Âge moyen } \\
\text { Expérience professionnelle moyenne } \\
\text { Ancienneté moyenne }\end{array}$ & $\begin{array}{l}40,1 \\
23,7 \\
10,4\end{array}$ & $\begin{array}{l}38,9 \\
20,7 \\
11,3\end{array}$ & $\begin{array}{l}40,4 \\
21,3 \\
12,4\end{array}$ & $\begin{array}{l}42,6 \\
22,3 \\
13,5\end{array}$ \\
\hline $\begin{array}{l}\text { Diplômes de formation initiale } \\
\text { Supérieur } \\
\text { Baccalauréat } \\
\text { Bepc-Bep-Cap } \\
\text { Cep } \\
\text { Aucun }\end{array}$ & $\begin{array}{r}7 \\
9 \\
25 \\
30 \\
29\end{array}$ & $\begin{array}{l}11 \\
11 \\
35 \\
22 \\
22\end{array}$ & $\begin{array}{l}16 \\
14 \\
38 \\
13 \\
19\end{array}$ & $\begin{array}{r}23 \\
15 \\
37 \\
7 \\
18\end{array}$ \\
\hline Taux de FPCE 1972-1977; 1980-1985; 1988-1993; 1998-2003 & $10,9 \%$ & $9,8 \%$ & $31,9 \%$ & $41,1 \%$ \\
\hline $\begin{array}{l}\text { Diplôme de FPCE oui } \\
\text { non }\end{array}$ & $\begin{array}{l}25,6 \\
74,4 \\
\end{array}$ & $\begin{array}{l}22,8 \\
77,2 \\
\end{array}$ & $\begin{array}{l}11,1 \\
88,9 \\
\end{array}$ & $\begin{array}{r}5,4 \\
94,6 \\
\end{array}$ \\
\hline $\begin{array}{l}\text { Durée de la FPCE: } \\
\text { Correspondance } \\
\text { Moins de } 41 \text { heures } \\
41-160 \text { heures } \\
161-320 \text { heures } \\
321-800 \text { heures } \\
\text { Plus de } 800 \text { heures }\end{array}$ & $\begin{array}{r}3,2 \\
18,1 \\
36,3 \\
17,6 \\
12,5 \\
12,2\end{array}$ & $\begin{array}{r}2,0 \\
26,8 \\
35,1 \\
14,4 \\
10,4 \\
11,2\end{array}$ & $\begin{array}{r}0,3 \\
53,3 \\
27,8 \\
5,9 \\
5,7 \\
6,9\end{array}$ & $\begin{array}{r}73,5 \\
15,9 \\
2,6 \\
3,5 \\
4,5\end{array}$ \\
\hline Durée moyenne de FPCE en heures & 309 & 280 & 244 & 111 \\
\hline Nombre total de FPCE & 0,38 & 2,17 & 2,15 & 2,66 \\
\hline
\end{tabular}

Sources: enquêtes FQP 1977, 1985, 1993 et 2003 de l'Insee.

Champ: salariés à la date de l'enquête qui étaient aussi salariés cinq avant.

individualisée, et de dépenses importantes de FPCE. Pour autant, ces dernières tendent à s'articuler à une gestion par les compétences dans laquelle on peut faire l'hypothèse que la formation continue tend d'une part à optimiser le travail en équipe et la polyvalence (FPCE courte d'adaptation) et, d'autre part, à retenir les meilleurs au sein de la firme au travers d'une valorisation conjointe de l'ancienneté et de la FPCE. On peut en effet supposer que les nouvelles formes organisationnelles définissent des capacités collectives spécifiques, que la firme ne veut pas perdre, d'autant plus qu'une bonne partie de son personnel a maintenant des diplômes de formation initiale potentiellement transférables. Pour autant, les mobilités sur le marché externe (d'emploi à emploi) sont conjointement extrêmement élevées dans cette période, ce qui suggère une compétition accrue entre les firmes pour la captation de la maind'œuvre jugée la plus performante (avec, évidemment, la valorisation salariale corrélative).

De même, la formation continue a considérablement évolué en vingt-cinq ans: le taux de formés a fortement augmenté, les diplômés ont chuté, et les formations courtes se sont multipliées. Rappelons que pour 1977 et 1985, la FPCE décrite est celle jugée par le salarié comme étant la plus importante, à l'inverse des deux autres années où il s'agit de la dernière formation suivie. Du coup, les taux d'accès (sur cinq ans), les durées et les diplômes ne sont pas strictement comparables pour 1977 et 1985 par rapport aux deux dates ultérieures. On peut avoir une idée des évolutions en pointant l'accès à une formation quelle que soit la date où elle a eu lieu (sauf pour 2003 où la FPCE n'est renseignée que sur les cinq dernières années, ce qui a impliqué de ne retenir que cet intervalle de cinq ans précédant la date des quatre enquêtes). Ainsi, $23 \%$ des salariés ont suivi une FPCE dans l'enquête de 1977, ils étaient 26\% en 1985 et $45 \%$ en 1993, soit un doublement (et non un triplement) entre 1977 et 1993 (à comparer avec l'accès sur les seules cinq dernières années, $c f$. tableau 1, où, sur des sources comparables, on note la permanence de ces tendances entre 1993 et 2003 , soit moins de diplôme et augmentation de 20 points des FPCE les plus courtes).

Dans une deuxième partie, on va s'intéresser aux évolutions des effets de la FPCE sur le salaire durant une période d'un quart de siècle, et, corrélativement, se pencher sur la valorisation de cette dernière en lien avec l'efficacité salariale de l'ancienneté. Dans une troisième partie, nous analyserons de manière plus globale les relations entre formation continue et les différentes mobilités au sein des marchés internes. En effet, autant l'an- 
cienneté ne renseigne que sur un stock (d'années) à l'intérieur de l'entreprise, autant il est indispensable d'analyser les rapports entre FPCE et les différentes mobilités sur le marché interne, qu'il s'agisse des mouvements en son sein (promotion, changement de poste) ou de l'accès à la firme (changement d'entreprise) où se trouve le salarié en 1993 ou 2003.

\section{Salaires et la formation professionnelle continue}

Il faut avant tout commencer par une question de méthode et de vocabulaire. On sait que certaines variables sont endogènes aux phénomènes que l'on veut analyser (en l'occurrence le salaire), telles que l'ancienneté dans l'entreprise (Lemistre, Plassard, 2002), ou la FPCE ( $c f$. surpa). L'idée est que les meilleurs sont mieux rémunérés, et que c'est cette propriété que l'on mesure au travers du fait de rester dans l'entreprise ou d'avoir une formation. Dans ces cas, il faut mettre en œuvre des procédures économétriques permettant de prendre en compte les biais d'hétérogénéité non observée afin de parvenir à «des effets nets » de la variable exogène utilisée (ancienneté et/ou FPCE). Si ces procédures sont apparemment les meilleures au plan technique, on peut s'interroger sur leur interprétation au plan économique. La recherche des «effets nets », pour légitime qu'elle soit, se conçoit dans une approche de type capital humain dans laquelle l'objectif est de mesurer l'apport additionnel d'un investissement donné (par exemple, une action de formation continue). On l'a vu, dans ce cadre les conséquences sur le salaire sont très faibles, voire nulles, ce qui n'est cependant pas le cas pour l'Allemagne où, après correction des biais, la formation continue conserve un effet propre important sur le salaire (BÉRET, DuPRAY, 2000a). Ce fait constitue, d'ailleurs, un indicateur intéressant des contextes sociétaux dans lesquels prennent place les formations initiales et continues. On ne voit cependant pas, dans le cas français, quel est l'intérêt du salarié à se former. Ce dernier pourrait simplement refuser la formation ou ménager son effort au cours de celle-ci. Mais il pourrait aussi, à l'issue de cette dernière, (menacer de) quitter la firme qui perdrait alors son investissement (dont la durée moyenne est de 111 heures en 2003, ce qui n'est pas rien). Pour que le «contrat» de formation soit réalisable, il faut donc qu'il réponde à l'exigence de participation entre les parties (l'utilité de l'agent doit s'accroître si son effort augmente BrousSEAU 1993), l'individu et la firme doivent tous deux bénéficier des effets de la FPCE. À l'inverse, si l'acceptation des biais d'hétérogénéité ne permet pas le calcul des effets nets, il correspond au contraire pleinement à une approche par le signal puisque, justement, ce dernier n'est qu'un indicateur indirect des capacités réelles (supposées) des personnes (on parle aussi de biais de sélection, ce qui constitue un vocable particulièrement judicieux ici). Dans cette optique, si l'on ne peut pas parler de rendement d'un investissement particulier, on peut parler d'effet, ou de marquage, de la FPCE sur le salaire dès lors que l'on enregistre une corrélation entre formation et rémunération (effet en ce sens que le marquage formatif fait que les salariés sont mieux, ou moins bien, payés que les autres).

On peut dégager plusieurs éléments avant d'en venir à l'impact de la FPCE (tableau 2). On observe tout d'abord une forte baisse des effets salariaux des diplômes de formation initiale entre 1977 et 1993, résultat qui se retrouve dans d'autres travaux (Baudelot, Glaude, 1989; Goux, Maurin, 1994). Ce phénomène, bien qu'atténué, se poursuit en 2003. Parallèlement, il est constaté une nette diminution des effets de l'ancienneté qui ne sont plus significatifs en 1993, malgré l'augmentation de l'ancienneté moyenne de deux ans, ce qui rejoint des résultats obtenus par ailleurs (GAUTIÉ 2004, Goux, Maurin, 1994). Certes, les effectifs de l'enquête 2003 sont plus faibles mais concernent cependant plus de 8000 salariés ( $c f$. tableau 1$)$. On peut utilement comparer ce résultat avec un autre travail similaire portant sur les salariés des enquêtes Emploi de l'Insee pour les années 1989 et 1984 et qui étaient aussi salariés deux ans avant. En retenant les années d'étude, l'expérience, l'ancienneté (et leurs carrés) pour les hommes, l'ancienneté avait un effet salarial positif en 1984 et négatif en 1989 (BÉret, 1992). Par contre, en 2003, l'effet de l'ancienneté est à un niveau élevé pour les raisons développées précédemment. On peut d'ailleurs noter que l'augmentation continue de l'ancienneté moyenne aux différentes dates n'est certainement pas étrangère à l'apparition de ce marché secondaire stable.

L'effet salarial lié à une FPCE est le plus fort pour 2003. Mais comme pour 1993, aussi bien le diplôme de FPCE que la durée de formation ne suivent pas une logique d'accumulation en capital humain, les diplômés et les formations longues n'ayant pas les effets les plus importants sur le salaire (tableau 2, modèles 2 et 3 ). Ces éléments confortent l'idée que la formation continue ne suit plus une logique d'accumulation en capital humain depuis les années 1990 mais, au contraire, renvoie à des éléments de marquage professionnel, encore accentués en 2003 dans la mesure ou, notamment, trois quarts des formations sont de très courte durée (contre la moitié en 1993) avec des effets sensiblement supérieurs à ceux de 1993. On va d'ailleurs s'intéresser maintenant à l'efficacité salariale de l'ancienneté pour montrer que, là aussi, l'accumulation de capital spécifique entraîne des effets plus forts pour les salariés auxquels a été octroyée une formation continue.

Il reste donc à mieux comprendre comment se construisent les capitaux professionnels sur le 
Tableau 2: Estimations des effets de la FPCE et de ses attributs, formation obtenue dans l'intervalle de cinq ans précédant les enquêtes

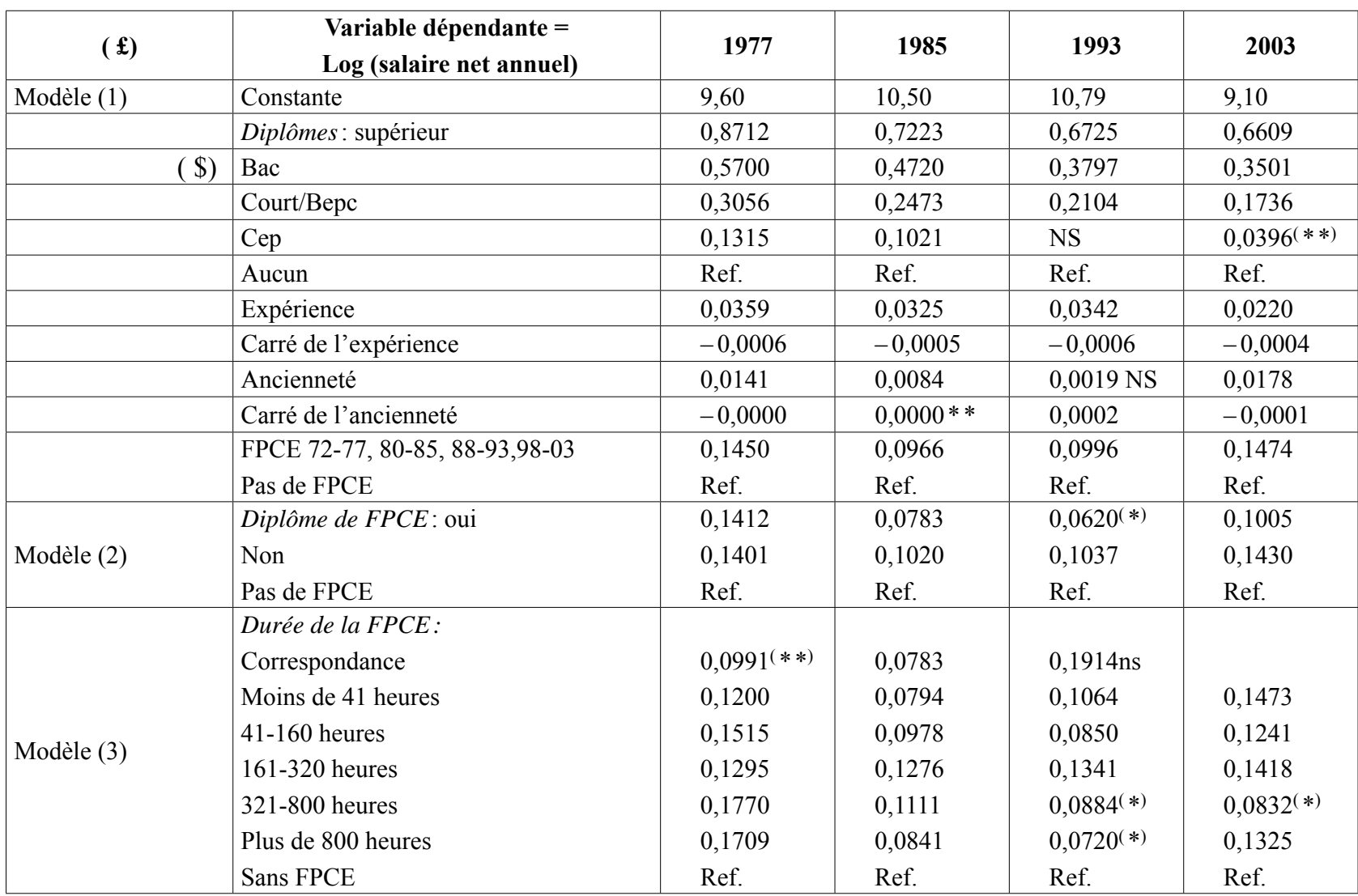

Sources : enquêtes FQP 1977, 1985, 1993 et 2003 de l'Insee.

( \$) : fonctions de gains diplôme - expérience - ancienneté - FPCE. Deux autres fonctions sont ensuite estimées en tenant compte successivement du diplôme, de la durée de FPCE. Il s'agit des FPCE terminées sur l'intervalle de cinq ans précédent la date des enquêtes.

Champ: salariés à la date de l'enquête qui étaient aussi salariés cinq avant.

(£): seuls sont présentés les coefficients de l'expérience, de l'ancienneté et du diplôme pour la première spécification.

Coefficients significatifs à $1 \%$ d'erreur, $(*)$ au plus à $5 \%,(* *)$ au plus à $10 \%$.

NS : non significatif.

Tableau 3 : Estimations de l'effet de l'ancienneté selon la FPCE

\begin{tabular}{|c|c|c|c|c|}
\hline Ancienneté & FPCE & 1985 & 1993 & 2003 \\
\hline En années & $\begin{array}{l}\text { Oui } \\
\text { Non }\end{array}$ & $\begin{array}{l}0,013 \\
0,008\end{array}$ & $\begin{array}{l}0,006 \\
0,001 \mathrm{NS}\end{array}$ & $\begin{array}{l}0,022 \\
0,016\end{array}$ \\
\hline $\begin{array}{l}\text { En classes: } \\
- \text { moins } 1 \text { an } \\
-1 \text { an moins de } 5 \text { ans } \\
-5 \text { ans moins de } 10 \text { ans } \\
-10 \text { ans et plus }\end{array}$ & OUI & $\begin{array}{l}0,107 \mathrm{NS} \\
0,054(*) \\
0,044(*) \\
0,099\end{array}$ & $\begin{array}{l}0,133 \\
0,110 \\
0,124 \\
0,079\end{array}$ & $\begin{array}{c}-0,106 \\
0,117 \\
0,040^{(* *)} \\
0,122\end{array}$ \\
\hline $\begin{array}{l}\text { moins } 1 \text { an } \\
-1 \text { an moins de } 5 \text { ans } \\
-5 \text { ans moins de } 10 \text { ans } \\
-10 \text { ans et plus }\end{array}$ & $\mathrm{NON}$ & $\begin{array}{c}-0,001 \mathrm{NS} \\
0,060 \\
0,039 \\
\text { Ref. }\end{array}$ & $\begin{array}{l}-0,050 \mathrm{NS} \\
-0,013 \mathrm{NS} \\
-0,002 \mathrm{NS} \\
\text { Ref. }\end{array}$ & $\begin{array}{l}-0,423 \\
-0,045^{(*)} \\
-0,072 \\
\quad \text { Ref. }\end{array}$ \\
\hline
\end{tabular}

Sources: FQP 1993 et 2003 de l'Insee. Résultats de deux fonctions de gains diplômes - expérience - ancienneté (*) FPCE.

Champ: salariés à la date de l'enquête qui étaient aussi salariés cinq avant.

Coefficients tous significatifs à $1 \%$ d'erreur, ${ }^{(*)}$ au plus à $\left.5 \%,{ }^{*}\right) 10 \%$.

NS : non significatif.

Note de lecture: en 2003, les salariés qui on suivi une FPCE et qui ont entre 1 ans et moins de 5 ans d'ancienneté ont un salaire sensiblement plus élevé que ceux qui ont 10 ans et plus d'ancienneté (ref.) et qui n'ont pas suivi de FPCE, au contraire, ceux qui ont une ancienneté de 1 à $<5$ ans mais qui n'ont pas suivi de FPCE ont un salaire plus bas que les plus anciens non formés. 
marché du travail, et notamment sur les marchés internes appréhendés, dans cette partie, du point de vue du temps passé dans la firme. On l'a vu, l'effet de l'ancienneté, qui était non significatif en 1993, est à son niveau le plus élevé en 2003. Est-ce à dire qu'en dix ans, il y a deux familles de capitaux spécifiques (au sens de G. Becker), l'apprentissage sur le tas d'un côté et la FPCE formelle de l'autre? Pour le savoir, ce qu'il faut analyser, c'est la manière dont se construit l'articulation de ces deux formes de constitution des capitaux professionnels. Dans un premier temps, on peut analyser globalement l'effet salarial de l'ancienneté selon que les salariés ont, ou n'ont pas, suivi une formation continue (tableau 3). Ce qui est frappant pour 1993, c'est que l'ancienneté a un effet positif sur le salaire pour les personnes qui ont eu une FPCE mais n'en a pas pour les non formés. De manière plus fine, l'ancienneté a un effet positif quelle que soit l'importance des années passées dans la même entreprise lorsqu'elles s'accompagnent d'une formation continue. À l'inverse, le marché interne ne joue plus aucun rôle, en 1993, lorsqu'il n'est pas redoublé par une FPCE, mais il retrouve ses attributs «classiques» en 2003, c'est-à-dire que les plus anciens non formés sont mieux rémunérés que les moins anciens non formés.

Autrement dit, c'est bien une logique de discrimination positive qui est à l'œuvre à travers l'octroi d'une formation continue dont la durée ne cesse de diminuer par ailleurs. On peut même avancer qu'en 2003, cette logique s'est renforcée du fait d'un assez fort impact salarial négatif de l'ancienneté pour la grosse moitié des salariés exclus de l'accès à une formation continue alors que cette dernière était non significative en 1993. Il faut attirer l'attention sur deux faits liés à ces bouleversements. Le premier est relatif aux tranches d'ancienneté retenues: moins de 10 ans en 2003 signifie que ces salariés ne sont pas (ou plus) dans les entreprises observées en 1993. Ces «nouveaux entrants» ce sont donc vus appliquer des règles de gestion internes qui ne prévalaient pas, ou peu, en 1993. Le deuxième fait est relatif au poids des marchés internes: en 1993 les salariés ayant 10 ans et plus d'ancienneté représentent $57,1 \%$ des cas contre $54,7 \%$ en 2003 alors que, parallèlement, on constate un accroissement de la durée de l'ancienneté moyenne: 10,4 ans en 1977, 11,3 ans en 1985, 12,4 ans en 1993 et 13,5 ans en 2003 ( $c f$. tableau 1). Cela se traduit par la forte augmentation des anciennetés longues. Ainsi, les salariés qui sont depuis 25 ans et plus dans la même entreprise ne sont que $11,5 \%$ en 1993 contre $18,6 \%$ en 2003. Cela signifie que les marchés internes en 2003 comptent à la fois plus d'entrants récents (moins de 10 ans) et beaucoup plus de très anciens (25 ans et plus).

\section{Mobilités, salaire et formations professionnelles continues}

Nous allons maintenant nous focaliser sur l'autre mécanisme de régulation des marchés internes, au-delà de la seule accumulation de temps, à savoir les différentes formes de mobilité. En effet, l'ancienneté que nous venons d'analyser n'informe pas sur les diverses mobilités internes qui ont eu lieu en articulation avec le fait de rester plus ou moins longtemps dans la firme. Tout d'abord, nous analyserons les effets salariaux liés aux modalités d'accès au poste occupé en 1993 et en 2003. Tout d'abord, il peut s'agir d'un poste unique, c'est-à-dire que le salarié n'a pas changé de poste depuis qu'il est dans cette entreprise. Si tel n'est pas le cas, il peut s'agir d'un changement de poste ou d'une promotion au sein de l'entreprise, quelle que soit la date où ces changements ont eu lieu. Par ailleurs, le poste occupé à l'enquête peut résulter d'un changement d'entreprise, en distinguant le cas où cette mobilité a eu lieu durant les cinq dernières années ou bien avant. Cela implique qu'à part les entrants durant l'intervalle de cinq ans, tous les autres salariés ont une ancienneté supérieure à cinq années. Dans un deuxième temps, nous verrons comment s'articulent ces changements avec la formation professionnelle continue en général, puis, de façon plus approfondie, en analysant l'ordre dans lequel ces évènements ont eu lieu. L'approche du capital humain laisse présumer une majorité de séquences «formation continue - promotion» et un gain maximal issu de cette combinaison dans la mesure où la promotion est susceptible de relever à la fois d'une fonction incitative motivant les efforts de formation consentis par les salariés et de l'objectif d'exploiter les investissements de manière optimale au travers de nouvelles affectations (PRENDERGAST, 1993).

On constate tout d'abord que les promotions et les changements de poste sont moins nombreux en 2003 (par exemple, pour les promotions, 9,2\% contre $11,7 \%$ en 1993 , tableau 4 , modèle 1 ), et que les changements d'entreprise sont de deux points supérieurs en 2003, ce qui rend bien compte des évolutions déjà notées dans les années 1990, avec la poursuite de la réduction des lignes hiérarchiques et la forte montée des mobilités emploi-emploi sur le marché externe.

Pour les deux enquêtes, on note l'importance des effets salariaux attachés au fait d'avoir bénéficié d'une promotion (tableau 4). Mais, de façon assez remarquable, c'est l'importance des effets en 2003, pour les promotions, mais aussi pour les salariés qui ont changé d'entreprise durant les 5 années qui précèdent le poste actuel, et, à un moindre degré, pour ceux qui ont changé de poste sur le marché interne. Autrement dit, le «marquage»salarial lié à ces mouvements est très sensiblement supérieur en 2003. 
Tableau 4 : Effet salarial de la formation continue et de la mobilité interne selon leur combinaison

\begin{tabular}{|c|c|c|c|c|}
\hline \multirow{2}{*}{$\begin{array}{l}\text { (\$) } \\
\text { Sur la fenêtre 1988-1993 et 1998-2003 }\end{array}$} & \multicolumn{2}{|c|}{ Effets } & \multicolumn{2}{|c|}{ Effectifs en \% } \\
\hline & 1993 & 2003 & 1993 & 2003 \\
\hline Modèle 1 & & & & \\
\hline Promotion & 20,7 & 32,7 & 11,7 & 9,2 \\
\hline Changement de poste & 5,1 & 16,2 & 12,0 & 11,1 \\
\hline Changement d'entreprise cinq dernières années & 11,1 & 29,1 & 15,2 & 15,3 \\
\hline Changement d'entreprise avant 1988 ou 1998 & ns & 8,6 & 18,1 & 20,0 \\
\hline Même poste & Ref. & Ref. & 43,0 & 44,4 \\
\hline Modèle 2 & & & & \\
\hline FPCE promotion (I) & 24,0 & 40,1 & 6,5 & 6,0 \\
\hline FPCE changement de poste (II) & 9,6 & 25,3 & 5,5 & 6,5 \\
\hline FPCE changement d'entreprise 5 ans (III) & 25,2 & 42,3 & 3,5 & 6,2 \\
\hline FPCE change d'entreprise avant 88 ou 98 (IV) & 10,7 & 21,1 & 3,7 & 6,7 \\
\hline FPCE et même poste $(*)$ & 8,6 & 13,4 & 12,8 & 17,5 \\
\hline Sans FPCE avec promotion $(*)$ & 23,1 & 35,6 & 5,3 & 3,2 \\
\hline Sans FPCE avec changement de poste $(*)$ & 6,5 & 17,4 & 6,5 & 4,6 \\
\hline Sans FPCE avec changement d'entreprise cinq ans $(*)$ & 9,4 & 27,3 & 11,7 & 9,1 \\
\hline Sans FPCE changement avant 1988 ou $1998\left(^{*}\right)$ & ns & 9,9 & 14,4 & 13,3 \\
\hline Sans FPCE et même poste $(*)$ & Ref. & Ref. & 30,2 & 26,8 \\
\hline $\begin{array}{l}\text { Modèle } 3 \\
\text { Décomposition des modalités (I) à (IV) ci-dessus : }\end{array}$ & & & \multicolumn{2}{|c|}{$\begin{array}{l}\text { Distribution des formé } \\
\text { qui ont connu un chan- } \\
\text { gement de poste depuis } \\
1988 \text { ou } 1998\end{array}$} \\
\hline FPCE puis promotion & 25,1 & 43,7 & 6,8 & 4,0 \\
\hline FPCE puis changement de poste & ns & 26,0 & 6,7 & 5,0 \\
\hline FPCE puis changement d'entreprise sur cinq ans & 27,0 & 35,5 & 6,4 & 5,3 \\
\hline Promotion puis FPCE & 28,7 & 39,2 & 18,0 & 14,0 \\
\hline Changement de poste puis FPCE & 10,1 & 24,2 & 16,3 & 15,7 \\
\hline Changement d'entreprise cinq ans puis FPCE & 24,9 & 43,2 & 9,1 & 14,2 \\
\hline Changement d'entreprise avant 1988 ou 1998 puis FPCE & 10,8 & 21,1 & 19,2 & 26,4 \\
\hline FPCE et promotion la même année & 13,7 & 39,9 & 8,9 & 5,3 \\
\hline FPCE changement de poste la même année & 13,6 & 28,4 & 5,7 & 5,0 \\
\hline FPCE changement d'entreprise cinq ans même année & 21,3 & 47,4 & 3,0 & 4,8 \\
\hline Sans FPCE et même poste & Ref. & Ref. & 100 & 100 \\
\hline
\end{tabular}

(\$): Sources FQP 1993 et FQP 2003 de l'Insee. Résultats de trois fonctions de gains diplômes - expérience - ancienneté - poste - FPCE.

Champ: salariés à la date de l'enquête qui étaient aussi salariés cinq avant.

Coefficients significatifs à $1 \%$ d'erreur ; ns : non significatifs à $10 \%$.

Lecture: en 1993, les salariés ayant eu une promotion ont un salaire plus élevé de (20,7) que ceux qui n'ont pas changé de poste (modèle 1), ce cas est porté à $(24,0)$ lorsque les promus ont suivi une FPCE par rapport a ceux qui n'ont ni changé de poste ni eu de FPCE (modèle 2), et atteint $(25,1)$ lorsque la FPCE a précédé la promotion (modèle 3).

* On n'analyse pas ces cas (sans FPCE et au même poste) dans le modèle 3, car ils donneraient les mêmes résultats que dans le modèle 2.

Le même constat prévaut lorsque l'on combine mouvements dans la firme et obtention d'une formation professionnelle continue. Ainsi, en 2003, aussi bien les promus formés que ceux qui viennent du marché externe et qui sont formés ont des rémunérations sensiblement plus élevées que les salariés non formés qui n'ont pas changé de poste (tableau 4, modèle 2). Corrélativement, même en l'absence de formation, ces deux types de mobilité sont liés à des salaires élevés, ce qui accrédite l'idée d'un renforcement des effets salariaux des mouvements dans les marchés internes. Au passage, on rejoint l'analyse effectuée du point de vue de l'ancienneté. Pour ceux qui viennent du marché externe durant l'in- tervalle retenu, cette dernière est par construction faible (cinq ans au plus). Dans le tableau 3, pour la classe 1 à $<5$ ans d'ancienneté, on voit l'effet positif sensible des salaires des formés et celui négatif pour les non formés.

Par ailleurs, il y a évidemment des rapports entre les mobilités et le fait d'avoir suivi une formation. Ainsi, en 1993, 55\% des promus ont eu au moins une FPCE dans les cinq ans (contre $30 \%$ pour ceux qui ont le même poste), on a respectivement $61 \%$ et $38 \%$ en 2003.

En outre, on dispose des années où ont eu lieu aussi bien les mobilités que les formations conti- 
Tableau 5: Durée de FPCE et nombre de formations selon les mobilités

\begin{tabular}{|c|c|c|c|c|}
\hline & \multicolumn{2}{|c|}{$\begin{array}{l}\text { Durées moyennes (heures) } \\
\text { de la FPCE obtenue entre } \\
1988-1993 \text { et } 1998-2003(\mu)\end{array}$} & \multicolumn{2}{|c|}{$\begin{array}{l}\text { Nombre total de FPCE ( } \$ \text { ) } \\
\text { (y compris celles avant } 1988 \\
\text { ou 1998) }\end{array}$} \\
\hline & 1993 & 2003 & 1993 & 2003 \\
\hline FPCE puis promotion & 361 & 158 & 5,09 & 4,30 \\
\hline FPCE puis changement de poste & 336 & 144 & 3,95 & 3,75 \\
\hline FPCE changement d'entreprise 5 ans & 359 & 232 & 3,10 & 3,31 \\
\hline Promotion puis FPCE & 109 & 81 & 6,36 & 6,88 \\
\hline Changement de poste puis FPCE & 118 & 72 & 6,13 & 6,49 \\
\hline Changement d'entreprise cinq ans puis FPCE & 86 & 94 & 4,88 & 4,83 \\
\hline Changement d'entreprise avant 1988 ou 1998 puis FPCE & 109 & 73 & 4,62 & 4,76 \\
\hline FPCE et même poste & 113 & 98 & 5,42 & 4,77 \\
\hline Sans FPCE avec promotion & - & - & 1,69 & 1,75 \\
\hline Sans FPCE avec changement poste & - & - & 1,19 & 1,30 \\
\hline Sans FPCE changement entreprise cinq ans & - & - & 0,35 & 0,84 \\
\hline Sans FPCE change entreprise avant 1988 ou 1998 & - & - & 0,39 & 0,65 \\
\hline Sans FPCE et même poste & - & - & 0,75 & 0,80 \\
\hline Ensemble & 244 & 111 & 2,15 & 2,66 \\
\hline
\end{tabular}

(\$): Sources FQP 1993 et FQP 2003 de l'Insee.

Champ: salariés à la date de l'enquête qui étaient aussi salariés cinq avant.

$(\mu)$ : calculées sur la base de la nomenclature en sept postes du questionnaire (cours par correspondance exclus).

(\$): depuis l'entrée en activité.

nues(14). Il est donc possible, dans presque $85 \%$ des cas, de reconstruire les séquences formationmobilités et mobilités-formation. Les attendus du capital humain sur la prédominance de l'enchaînement formation-mouvement avaient déjà été battus en brèche pour 1993 (BÉRET, DuPRAY, 1998). Cette tendance s'est encore accentuée en 2003 (tableau 4, modèle 3). Ainsi, en 1993, la formation précédait une mobilité (promotion, changement de poste et changement de firme) dans $19,9 \%$ des cas, tandis que la formation suivait une mobilité pour $43,4 \%$, tandis qu'en 2003 on a respectivement $14,3 \%$ et $43,9 \%$.

Pour ce qui est des effets, en 2003 la séquence formative, qu'elle précède ou succède aux mobilités, n'entraîne pas de différences flagrantes. Cependant, autant pour 1993 que pour 2003, les formations qui suivent une mobilité sont sensiblement plus courtes (tableau 5), renforçant l'aspect signalement attaché aux FPCE. Parallèlement, c'est aussi pour les séquences mobilités-formation que le nombre de FPCE suivi est le plus élevé renvoyant, à nouveau, à la dimension marquage plutôt qu'investissement de la formation.

Il convient aussi de souligner certaines caractéristiques du recours au marché externe. Ainsi, la

(14) Pour 1993, on ne dispose pas du mois d'obtention du poste actuel, on doit donc se contenter de l'année pour les deux enquêtes. séquence FPCE - changement d'entreprise (dans les cinq ans) informe que la (dernière) formation continue a eu lieu chez l'employeur précédent. Aux deux dates, les effets sont quasiment les plus forts, les FPCE sont les plus longues, tandis que le nombre de formation suivi est le plus faible parmi les salariés ayant eu une formation durant les cinq ans. À l'inverse, les salariés dont la dernière formation a eu lieu dans l'entreprise après un changement d'employeur ont des durées faibles mais ont suivi plus de 1,5 FPCE de plus, ce qui laisse augurer qu'ils ont probablement suivi des formations (peutêtre plus longues) dans la firme antérieure. Dans les deux cas, ceux qui ont changé d'entreprise durant les cinq dernières années sont les plus jeunes $(35,6$ ans en moyenne contre 40,4 ans pour l'ensemble en 1993, et respectivement 37,5 et 42,6 en 2003). Ils ont aussi une autre particularité : en 2003 (on ne dispose pas de l'information pour 1993), le changement d'entreprise s'est réalisé suite à une démission pour $59 \%$ des mobiles (et $27 \%$ lors d'une fin de contrat et $11 \%$ suite à un licenciement), ce qui est tout à fait considérable(15) (par exemple, dans

(15) La sélection de l'échantillon implique que les personnes soient salariées à la date de l'enquête et cinq ans avant. Ce faisant, on ne retient que les individus les plus employables dont le premier emploi date d'au moins cinq ans. L'analyse porte donc sur le cœur des marchés internes dont on peut étudier les transformations au-delà des variations dans le temps, aussi bien de certaines formes particulières d'emploi que de la conjoncture lors des premières années de vie active des jeunes. 
les établissements de 50 salariés ou plus, les sorties suite à une démission représentent autour de $20 \%$ en 2001 et 2002 et les fins de contrat autour de $70 \%$, (Agodor et al., 2004) et conforte un marquage positif pour l'entreprise d'accueil.

Par ailleurs, on peut s'interroger sur l'influence relative de la dernière formation et du nombre total de FPCE suivies depuis le début de la vie active. Pour 1993, on avait montré qu'en tenant compte de la date de fin des FPCE et du nombre de FPCE, seules les formations antérieures à 1998 avaient un effet positif(16), suggérant que c'était l'enchaînement de formations qui faisait avant tout valeur alors que ce n'était pas le cas en 1977 (BÉRET, DuPrAY 1998). Lorsque l'on introduit le nombre de FPCE au modèle 2 du tableau 4 , on retrouve ce phénomène pour les formés dans les cinq dernières années en 1993. Le nombre de FPCE a un effet élevé $(2,7 \%$ par formation) avec, corrélativement, une division par deux de l'effet élevé attaché à une promotion ou à un changement d'entreprise récent.

En revanche, en 2003, l'efficacité économique du nombre de FPCE suivies est faible $(1,2 \%)$, et les effets de la dernière formation sur les cinq dernières années (en lien avec les mouvements : promotion...) ne sont que très peu modifiés. Ceci suggère que le «marquage» formation continue retrouve une traduction salariale élevée, au-delà de l'ensemble des épisodes formatifs (pour laquelle on peut cependant s'interroger sur sa nature d'investissement au vu de la brièveté de sa durée moyenne et de l'absence de conséquences claires en fonction de son volume). De manière plus générale, cela signifie qu'en 1993, c'est avant tout la récurrence des FPCE qui traduit un marquage salarialement observable. Au contraire, en 2003, le FPCE est utilisée conjointement pour optimiser le travail en équipe (durées de formation faibles) et fidéliser les ressources spécifiques qui découlent des nouvelles formes organisationnelles. Dans ce cadre, la dernière FPCE conserve un marquage salarial propre au-delà de l'ensemble des formations suivies durant l'ensemble de la vie active (les salariés n'ayant pas eu de formation durant les cinq dernières années en ont d'ailleurs fort peu eu avant, $c f$. tableau 5).
En un quart de siècle, en lien avec les transformations sur les marchés du travail, la formation professionnelle continue s'est considérablement transformée, aussi bien du point de vue de ses caractéristiques que de ses conséquences salariales. En ne considérant que les cinq années précédant les enquêtes pour 1977, 1985, 1993 et 2003, le taux de formés a explosé, la certification s'est effondrée et les durées ont fondu. De même, les conséquences liées à la conception de la formation comme investissement n'ont plus lieu d'être puisque diplôme et durée des FPCE n'ont plus d'effets allant dans ce sens. L'ancienneté dans la firme, dont l'effet n'était plus significatif en 1993, le redevient en 2003, suite aux transformations relatives aux marchés du travail dans les années 1990, mais seulement pour les salariés à qui l'entreprise a octroyé une formation continue, bien que cette dernière soit de faible durée (moins de 41 heures pour les trois quarts d'entre eux). Cette articulation singulière entre apprentissage sur le tas long (13,5 ans en moyenne) et FPCE courte laisse augurer de nouvelles articulations dans la construction des compétences et des rémunérations dans les marchés internes.

Ce statut de la FPCE se combine avec des mobilités jouant un rôle similaire de discrimination positive. La formation intervient d'ailleurs le plus souvent après les mobilités, tout en étant très sensiblement plus courte, ce qui confirme le fait qu'elle ne fait que sanctionner, après coup, des compétences acquises antérieurement dans le système scolaire ou dans les années d'expérience passées. Deux faits marquants distinguent 1993 et 2003. Le premier est l'importance des gains salariaux liée aux mobilités en 2003, et ce, d'autant plus qu'elles s'accompagnent d'une formation continue. Le deuxième est relatif à l'articulation entre dernière formation et nombre de FPCE durant la vie active, ce dernier jouait un fort rôle en 1993, au point d'annuler les effets de la formation renseignée, sa place est plus faible en 2003 à l'avantage de mobilités sur les marchés internes dont les conséquences économiques pour les salariés sont très sensiblement plus fortes.

(16) Pour 2003, la dernière formation suivie n'est informée que si elle se trouve dans l'intervalle de cinq ans qui précède l'enquête, on a donc aussi uniquement retenu ce dernier pour 1993 comme dans l'ensemble du texte. 


\section{Bibliographie}

Agodor S., Grcic S., Rouat I. (2004), «Les mouvements de main-d'œuvre dans les établissements de 50 salariés ou plus en 2001 et $2002 »$, Insee Résultats Société n 27.

Amossé T. (2002), «Vingt-cinq ans de transformation des mobilités sur le marché du travail», Données sociales, Insee.

Aventur F., MöBus M. (1996), «La formation continue dans les entreprises: la place de la France en Europe», Bref, $\mathrm{n}^{\circ} 116$, Cereq.

Barreau J., Brochard D. (2003), «Les politiques de rémunération des entreprises: écarts entre pratiques et discours », Travail et Emploi, ${ }^{\circ}$ 93, Dares.

Baudelot C., Glaude M (1989), «Les diplômes se dévaluent-ils en se multipliant?», Économie et Statistique, $\mathrm{n}^{\circ} 225$, Insee.

Bentabet E., Gauthier C., Marion I. (2001), La formation professionnelle fiancée par les entreprises, Documents $n^{\circ} 160$, Cereq.

Béret P., Lewandowski M. (2007), «Politiques de ressources humaines et salaires: compétences et VAE», in J.-M. Lattes, P. Lemistre, P. Roussel, Individualisation des salaires et rémunération des compétences, Paris, Economica.

BÉRET P., Dupray A. (2000a), «Allocation et effet salarial de la formation professionnelle continue en France et en Allemagne: une approche en terme d'information», Économie Publique, $\mathrm{n}^{\circ} 5$.

BÉRET P., Dupray A. (2000b), «Qualifications et valorisation salariale de la formation continue en France», in V. Vandenberghe (Eds), Formation professionnelle continue: transformations, contraintes et enjeux, Académia Bruylant.

Béret P., Dupray A. (1998), «La formation professionnelle continue: de l'accumulation de compétences à la validation de la performance », Formation Emploi, $\mathrm{n}^{\circ} 63$, Cereq.

BÉRET P. (1992) «Salaires et marchés internes: quelques évolutions récentes en France», Économie appliquée, vol. XLV, $\mathrm{n}^{\circ} 2$.

Berton F., Podevin G. (1991), «Vingt ans de formation professionnelle continue: de la promotion sociale à la gestion de l'emploi», Formation Emploi, n ${ }^{\circ} 34$, Cereq.

BRIZARD A. (2004), 1999-2002: des hausses de salaires de moins en moins individualisées, Premières Synthèses, $n^{\circ}$ 22-1, Dares.

Brousseau E. (1993), L'économie des contrats. Technologies de l'information et coordination interentreprises, Paris, PUF.

Crocquey E. (1995), «La formation professionnelle continue: des inégalités d'accès et des effets sur la carrière peu importants à court terme». Travail et Emploi, $\mathrm{n}^{\circ} 65$, Dares.

Dubar C., Podevin G. (1990), «Formation et promotion en France depuis vingt ans », Bref, $n^{\circ} 59$, Cereq.

Depardieu D. Payen J.-F. (1986), «Disparités de salaire dans l'industrie en France et en Allemagne: des ressemblances frappantes», Économie et Statistique, $\mathrm{n}^{\circ} 188$, Insee.

Dupray A., Hanchane S. (2003), «Modalités de participation à la formation continue et effets sur la carrière salariale», in Regards pluriels sur la formation continue, De Boeck.

Eyraud F., Marsden D., Silvestre J.J. (1990), «Marché professionnel et marché interne du travail en GrandeBretagne et en France», Revue internationale du travail, $n^{\circ} 4$.

Fougère D., Goux D., Maurin E. (2001), «Formation continue et carrières salariales: une évaluation sur données individuelles», Annales d'économie et de statistique, $\mathrm{n}^{\circ} 62$.

FouRNIER C. (2003), «Développer la formation des "seniors"? Deux questions préliminaires, Formation Emploi, $\mathrm{n}^{\circ} 81$, Cereq.

FourNiER C. (2001), «Hommes et femmes salariés face à la formation continue $»$, Bref, $\mathrm{n}^{\circ} 179$, Cereq.

Fournier C., Hanchane S., Lambert M., Perez C., Théry M., Thomas G. (2001), «Un panorama de la formation continue des personnes en France», Bref, $\mathrm{n}^{\circ}$ 172, Cereq.

Gautié J. (2004), «Les marchés internes du travail, l'emploi et les salaires», Revue française d'économie, $\mathrm{n}^{\circ} 4$.

Gadéa C., Trancart D. (2003), «Pratiques de formation continue et promotion au statut de cadre: un lien paradoxal», Formation Emploi, n ${ }^{\circ}$ 81, Cereq.

GÉLOT D. (2006), «Le rôle de l'encadrement intermédiaire dans la formation en entreprise», Travail et Emploi, $n^{\circ} 107$, Dares.

GÉLOT D., MinNi C. (2002), «Formation continue et insertion: un taux d'accès élevé et des formations qualifiantes après la fin des études", Premiers Résultats, Premières Synthèses, $\mathrm{n}^{\circ} 10.2$, Dares.

Germe J.-F. (2001), «Au-delà des marchés internes: quelles mobilités, quelles trajectoires?», Formation Emploi, $\mathrm{n}^{\circ} 76$, Cereq.

Goux D., Maurin E. (1997), «Les entreprises, les salariés et la formation continue», Économie et Statistique, $\mathrm{n}^{\circ} 6$, Insee.

Goux D., Maurin E. (1994), «Éducation, expérience et salaires: tendances récentes et évolutions de long terme», Économie et Prévision, $\mathrm{n}^{\circ} 5$. 
Goux D. (1991), «Coup de frein sur les carrières », Économie et Statistique, $\mathrm{n}^{\circ} 249$, Insee.

Hanchane S., LAmbert M. (2003), «La variété des modes de formation: usages et enjeux», Formation Emploi, $\mathrm{n}^{\circ} 81$, Cereq.

Lattes J.-M., Lemistre P., Roussel P. (2007), Individualisation des salaires et rémunération des compétences, Paris, Economica.

Lemistre P., Plassard J.-M. (2002), « Stratégies de mobilité et rendements de l'ancienneté en France», Économie et Prévision, $\mathrm{n}^{\circ} 155$.

Maurice M., Sellier F., Silvestre J.-J. (1982), Politique d'éducation et organisations industrielles en France et en Allemagne, Paris, PUF.

Méhaut P. (1996), «Se former tout au long de la vie?», Bref, $\mathrm{n}^{\circ} 120$, Cereq.

Mincer J., Jovanovic B. (1981), Labor mobility and wages. In Rosen S. (ed.), Studies in labor markets, Chicago University Press.

Perez C., Thomas G. (2006), «Trajectoires précaires d'emploi et formation continue», Économie et Statistique, $\mathrm{n}^{\circ} 388-389$, Insee.
Perez C. (2003), «La formation des agents de la fonction publique au miroir du privé», Formation Emploi, ${ }^{\circ} 81$, Cereq.

Petit H. (2003), «Les déterminants de la mise en œuvre d'un mode de gestion de l'emploi», Économie et Statistique, $\mathrm{n}^{\circ} 361$, Insee.

Prendergast C. (1993), "The rôle of promotion in inducing specific human capital acquisition”, Quarterly Journal of Economics, Vol. 108

Théry M., Rousset P., Zygmunt C. (2002), «L'Europe de la formation tout au long de la vie reste à construire», Bref, $\mathrm{n}^{\circ} 187$, Cereq.

Silvestre J.-J. (1986), Marchés du travail et crise économique: de la mobilité à la flexibilité, FormationEmploi, ${ }^{\circ} 14$, Cereq.

Trautmann J. (2003) «Accès et retours à la formation», Formation Emploi, $\mathrm{n}^{\circ}$ 81, Cereq.

VAlette A. (2007), «Systèmes d'emploi français et britannique: évolutions entre 1980 et $2001 »$, Économies et Sociétés, $\mathrm{n}^{\circ} 12$.

Verdier E. (1995), «Politique de formation des jeunes et marché du travail : la France des années $80 »$, FormationEmploi, $\mathrm{n}^{\circ}$ 50, Cereq. 